\title{
Expectations About Counseling for a Mental Illness Among Primary School Teacher Trainees in Kenya
}

\author{
Eunice Jemalel Nyavanga ${ }^{1,2, *}$, Maurice Barasa ${ }^{3}$ \\ ${ }^{1}$ Department of Social and Human Development, Faculty of Social Sciences and Technology, the Technical University of Kenya, Nairobi, \\ Kenya \\ ${ }^{2}$ Malel Bibwob Clinical Psychologists Consultancy (MICAP), Nairobi, Kenya \\ ${ }^{3}$ M\&E, International Planned Parenthood Federation - Africa Region, Nairobi, Kenya
}

Email address:

enyavanga@yahoo.com (E. J. Nyavanga),barazam@gmail.com (M. Barasa)

${ }^{*}$ Corresponding author

\section{To cite this article:}

Eunice Jemalel Nyavanga, Maurice Barasa. Expectations About Counseling for a Mental Illness Among Primary School Teacher Trainees in Kenya. Science Journal of Public Health. Vol. 4, No. 4, 2016, pp. 305-311. doi: 10.11648/j.sjph.20160404.16

Received: May 31, 2016; Accepted: June 12, 2016; Published: June 23, 2016

\begin{abstract}
The objective of this study is to determine and document expectations about counseling for a mental illness among public primary teacher trainees in Kenya. Self-administered demographic questionnaire and opinions about mental illness scale brief form (EAC-BF) were presented to the participants. The EAC-BF consisted of three factors of personal commitment, facilitative conditions and counselor expertise. The ethical protocol was followed from getting authority from the Ethics Board to informed consent from the participants. Out of the 2925 questionnaires presented, 2777 were returned fully filled, a return rate of $94.34 \%$. Summative scores indicated moderate (between 2.34 and 4.67 ) towards negative expectations about counseling, with significant differences in year of study, gender, marital status, or ever taught before coming to college. There was a correlation between all the EAC factors. This study found more negative expectations about counseling for a mental illness and recommended on an intervention to improve expectations towards counseling.
\end{abstract}

Keywords: Mental Illness, Expectations, Counseling

\section{Introduction and Background}

Mental disorders are common in the general population and have an early age onset (between 14-24years) that has been associated with adverse societal costs, [1]. Mental health problems including substance abuse are leading in years of lost life globally (YLD). Further more they have been found increasing especially among individuals between 10-29 years lately, [2]. In addition, Whiteford et al [2], found out that there was an increase of mental health problems including substance abuse by $37.6 \%$ between 1990 and 2010 . Poor mental health is associated with poorer educational achievements, other health and developmental concerns, substance abuse, and sexual health. Further more mental health problems of the youth are a crucial public health concern in order for them to be able to fulfill not only their potential but contribute fully to the development of their communities [3], and achieve the Sustainable Development
Goals (SDG), set by the United Nations in 2015 to be achieved by 2030 [4]. Research has identified Africa to have the greatest rate of disability-adjusted life-years (DALYS), at 2.5 times greater than the developed countries, [5].

Mental Health professionals are becoming aware of the necessity to support their activities using practice-based scientific research. The reasoning behind this is that results of such research will benefit the quality of treatments, as well as subsequent knowledge about psychiatric illnesses and the effectiveness and efficiency of the intervention programs. The use of psychological treatment has been found to have better outcomes compared to other treatments, specifically pharmacotherapy alone. [6]. Although mental disorders develop at adolescence and early adulthood, evidence has also indicated that this population does not seek counseling, [7]. Seeking counseling at this stage would reduce severity at a later age and reduce the development of secondary disorders. [8] 


\subsection{Background}

Expectations about counseling refer to an individual's belief in the outcome of counseling. This includes the characteristics of the counselor and the amount of disclosure they are expected to do in a counseling session. Expectations about counseling influence the success of therapy in terms of their decisions to enter into therapy and remain in therapy. Research has further indicated that negative expectations of counseling is a barrier to attitudes towards seeking professional psychological help, and detrimental to the counseling process. [9]

Counseling is used specifically for patients who are motivated to manage their own illnesses in order to develop skills and supports to control symptoms and pursue behavior change $[10,11]$. Counseling can take the form of individual, group, family or a combination. Although several studies in Expectations about Counseling among college students and other population have been done, none has been done among public primary school teachers and Kenya.

\subsection{Teacher Training in Kenya}

All students admitted to teacher training colleges must have completed at least four years of secondary education and hold the Kenyan Certificate of Secondary Education (KCSE) of a Grade C or better. The teacher training course lasts two years, at the end of which students are awarded a primary one (P1) Certificate, although this depends on their success in the centrally set examinations by the Kenya National Examination Council (KNEC).

The primary school Teacher Education course takes two years and students study ten subjects in the first year and nine subjects in the second year. The current 8-4-4 Primary Teacher Education (PTE) curriculum was first introduced in 1986 and was later revised in 1994. It was recently revised again in 2004 following the revision of the primary school curriculum reforms, so as to bring the two curricula into harmony. The revised curriculum incorporates aspects of Information and Communication Technology (ICT), HIV/AIDS, Special Education, Open Learning and Distance Education (OL\&DE). This curriculum does not have any training in mental health, [12].

\section{Methodology and Study Design}

\subsection{Methods}

\subsubsection{Settings and Study Population}

Convenience sampling was used to identify four colleges out of twenty public primary teachers colleges in Kenya. It was also established that these trainees were recruited from all over the country. Based on lists from the Ministry of Education enrollment (Statistics Department) the effective population for the study was estimated at 3, 400 (out of 17, 000) trainees from the sampled colleges. The colleges sampled included Kilimambogo, Murang'a, Thogoto and Machakos. This study is part of a larger longitudinal quasi- experimental (intervention) study; "The Effects of Psychoeducation on Mental Health Consultation Attitudes among Primary School Teacher Trainees in Kenya".

All consenting participants from the identified colleges were recruited for the study to increase the generalizability of the findings. The author assumed that primary teacher trainees would understand the working in the EAC scale and would be honest and accurate in their responses to the questionnaire.

2,925 questionnaires were presented to the participants, and 2,777 were returned fully filled, a response rate of $94.34 \%$.

\subsubsection{Ethical Considerations}

The Kenyatta National Hospital Ethics Committee (KNHEC) which sets forth research ethics concerning individual's data in Kenya approved the protocol while permission was provided by Ministry of Education (Research Department) and written informed consent was obtained from the college principals and the participants. The college principals were given a clear explanation of the purpose, plan and implication of the study. They were also informed that participation was voluntary and with no intrusive procedures and if they felt emotional they would see the college counselor or the team of research assistants or the researchers whose contacts were also provided.

\subsubsection{Study Procedures and Instrumentation}

The teacher trainees were presented with a selfadministered demographic questionnaire and standardized tool, Expectations about Counseling Brief Scale. The demographic questionnaire asked teachers on their year of study, age, gender, religion, marital status, whether they grew up in a village or an urban setting and whether they taught before coming to college.

The Expectations About Counseling-Brief Form (EAC-B; H. E. A. Tinsley

To measure expectations about counseling, the Expectations about Counseling - Brief Form (EAC-B) was used. This scale was first developed in 1980 as a 153 -item questionnaire to measure students' expectations of counseling. Tinsley [13] later developed a brief version of the questionnaire, the EAC-B as a briefer alternative to the original Expectations about Counseling developed by Tinsley et al, [14]. Because of the correlations between scores on the full and brief versions of the EAC are high, $(\mathrm{r}=0.85)$, Tinsley recommended that researchers use the brief version for most research applications. This researcher requested for permission to use this tool, and Tinsley personally wrote back to grant the permission to use the tool and provided the scoring scale. The scale consists of 66 items that assess the constructs about which help seekers might have expectations. The 66 items were answered on a seven-point Likert-type scale, with response options that range from not true (1) to definitely true (7). The 66 items are grouped into 17 scales that examine various expectations about the various expectations about the counseling process. The 17 scales measure three factors that have been replicated across 
numerous studies, $[14,15]$.

The EAC-B measures expectancies about client and counselor attitudes and behaviors, counselor characteristics, and counseling process and outcome. Summing the responses to the items assigned to each and dividing by the number of items calculated scale scores on the EAC-BF. The scale score for motivation, for example, can be obtained by summing the responses to items 14,15 , and 18 and dividing by 3 ; the scale score for responsibility is the sum responses to items $8,9,29$, and 30 , divide by four. Once the scale scores are calculated, scores on the first three factors reported by Tinsley et al [15] were obtained by adding the scale scores indicated below and dividing by the number of scale scores indicated, Scales are calculated by summing the responses to the items assigned to several smaller subscales, and dividing these subscales into respective categories.

This instrument measures expectations in three areas (a) Personal Commitment, (b) Facilitative Conditions, and (c) Counselor Expertise, created by Hatchett and Han [16].

(a) Personal Commitment factor: This factor is made up of twenty-two (22) items subdivided into seven (7) subscales. The subscales include the sub-factors of Responsibility $(8,9$, 29, \& 30), Openness $(5,19,25)$, Motivation $(14,15,18)$, Attractiveness (2, 11, 17), Immediacy (4, 10, 12), Concreteness $(33,37,41)$ and Outcome $(13,23,28)$, which measures respondents' expectations about assuming personal responsibility for working hard and achieving progress in counseling. This factor measures the expectations to assume personal responsibility for working hard and achieving progress in counseling, [15]. This reflects the subjects' expectations about what they will have to invest in counseling, the amount of personal responsibility the client expects to assume in counseling. Scores for this factor range from 22 to 154. Extremely high scores on this factor represent perfectionist thinking or a tendency towards compulsiveness in that they depict an unrealistically high level of responsibility. Low scores reveal a naïve belief that the client bears no responsibility for the success of counseling.

The personal commitment factor sub-scales describe the following. (1) Responsibility -measures the extent to which the client expects to make his or her own decisions, talk about concerns, work on concerns outside the counseling process, and be responsible for outcomes (2) Openness measures the extent to which the client expects to express and discuss his or her emotions and how much he or she feels doing so (3) Motivation-measures the extent to which the client expects to remain in counseling even if he or she is uncertain if it will help or if it is unpleasant (4) Attractiveness - measures the extent to which the client expects to like the counselor (5) Immediacy - measures the extent to which the client expects to gain experience in new ways of solving problems and relate openly and honestly to another person within the counseling relationship (6) Concreteness -measures the extent to which the counseling process is expected to help the client identify and solve problems as well as identify and understand his or her feelings, problem situation and behaviour (7) Outcome measures the extent to which the counseling process is expected to help the client gain better self-understanding, become better, able to help his or herself in the future and improve relationships with others

(b) The Facilitative Conditions factor made up of twentyone (21) items consisting seven (7) sub-scales comprised of the Acceptance $(46,59,61)$, Confrontation (56, 62, 64), Genuineness (40, 44, 53), Trustworthiness (42, 45, 60), Tolerance $(58,63,66)$, Nurturance $(38,49,55)$, and SelfDisclosure $(48,54,57)$. This factor measures the expectations that the interview conditions identified as theoretically necessary for progress in counseling will be present. Scores in this factor may range from 21 to 147 . High scores on this factor represent a degree of idealism that counselors are unlikely to attain, whereas low scores represent pessimism, skepticism, or cynicism about what counseling will be like.

The Facilitative Conditions factor subscales measurements are described as follows: (1) Acceptance - The extent to which the counselor is expected to be warm and friendly; (2) Confrontation - measures the extent to which the counselor is expected to challenge discrepancies between what the client wants and how she or he behaves; (3) Genuineness measures the extent to which the counselor is expected, to be honest, real and respectful; (4) Trustworthiness -measures the extent to which the counselor is expected to inspire confidence and trust; (5) Tolerance - measures extent to which the client expects the counselor to be calm and easy going; (6) Nurturance - measures the extent to which the counselor is expected to provide encouragement, reassurance, support, and praise; (7) Self-Disclosure - The extent to which the client expects the counselor to discuss his or her own problems and attitudes and relate them to the clients problems.

(c) Finally, the Counselor Expertise factor made up nine (9) items consisting of three (3) sub-scales which include Directiveness (32, 34, 43), Empathy (35, 39, 51), and Expertise $(36,47,50)$. This factor measures the expectation that the counselor will be a skilled practitioner who will be capable of helping the client. Scores in this factor may range from 9 to 63 . High scores indicate magical thinking in that the counselor is viewed as having tremendous curative powers. Low scores indicate a pessimistic or perhaps even fatalistic expectation that there is no way the counselor will be able to help the client.

The Counselor Expertise factor subscales measurements are described as follows: (1) Directiveness - measures the extent to which the client expects the counselor to offer direct advice; (2) Empathy - measures the extent to which the client expects the counselor to understand how he or she feels; (3) Expertise - measures the extent to which the counselor is expected to know how to help the client solve his or her problems, and to be able to determine what the problem is.

High scores on each of the three factors correspond to positive or optimistic counseling expectations in each 
domain. Overall, the internal reliability of the scales of the EAC-B ranges from 0.69 to 0.82 with a median reliability of 0.77 . Test-retest reliabilities over a period of 2 months ranged from 0.47 to 0.87 , with a median reliability of 0.71 [15]. The realism scale is the most experimental scale. According to Tinsley et al [13], the investigator must score to reflect the local situation in order to obtain meaningful results. He further indicated that these scales should be judged against local practices. The validity of this scale is uncertain, Tinsley et al [13].

The EAC-BF is the most widely used measure of expectations in counseling research, which has been extensively used in the area of psychotherapy research [16]. Other studies have reported good construct validity for the EAC-B and its scales [16]. The brief form has been reported to correlate well with the long form $(0.78$ to 0.95$)$ and also to be more highly related to external validity criteria [16].

Although some studies have used the four-factor model of the EAC-BF, some research has suggested that a three-factor model might provide a better description of the factor structure of the EAC-BF $[16,17]$. This study used the three main factors and the realism scale. Summing the responses to the items and dividing the summed score by the number of items in the respective scales obtained scale scores. Summing the relevant scale scores and dividing by the number of scales in that factor obtained the three-factor scores.

The instructions for EAC-B asked respondents to imagine that they were about to see a college counselor or psychologist for their first interview and that we would like to know what they think counseling will be like, and to report their expectations regarding their imagined counseling experience. For each statement, they were asked to indicate what they expect counseling to be like using the rating scale printed at the top of the questionnaire. They were also asked to record their ratings of the statements on the answer sheet provided by filling in the cycle corresponding to the number that most acutely reflects their expectation. They were also asked not to make any marks in the questionnaire booklet, to answer each question as quickly and accurately as possible, and to finish each page before going to the next. Instructions also required them not putting their name on the answer sheet because responses would be kept in the strictest confidence. They were also informed that their answers would be combined with the answers of others like them and reported only in the form of group averages.

\subsection{Data Management and Analysis}

The collected data for the study were analyzed using the statistical Packages for the Social Software (SPSS), version 19.0. This analysis included descriptive statistics, t-test (independent sample), factor analysis of variance (MANOVA) and summary statistics. Descriptive statistics such as cross-tabulation, measures of central tendency (mean) and measures of variability (range and standard deviation) were used to describe the data. The t-test (a parametric statistics) was used to determine the significance difference, a probability level of $\mathrm{P}<0.05$. The Multiple Analysis of Variance (MANOVA); a statistical procedure use to assess group differences across multiple dependent variables simultaneously was used based on the independent variables of age, gender, year of study, religion, marital status, whether they grew up in a village or an urban setting and whether taught before or not.

\section{Results}

Out of the 2777 participants, 1466 (52.8\%) were female, $605(21.8 \%)$ were 20 years of age and under, $1664(59.9 \%)$ were between 21 and 25 years of age, and 508 (18.3\%) were over 25 years of age. $1274(45.9 \%)$ were first years, 597 $(21.5 \%)$ were married, $2(0.1 \%)$ were widowed, $21(0.8 \%)$ were divorced or separated and $2157(77.7 \%)$ were not married. Those who were Christians were 2665 (96.0\%), while those who were Muslims were $82(3.0 \%)$ and others were $29(1.0 \%)$. Those who had taught before coming to college were $1171(42.2 \%)$.

\subsection{Summative Scores for EAC-BF per Demographic Characteristics of Participants}

The mean summative score of expectation ranges between 1 and 7, with higher score implying positive opinion. A score of less than 2.33 implies a negative level of expectation; between 2.34 and 4.67 implies a moderate level of expectation while a score of 4.68 and above implies a positive expectation. The results among these participants indicate a moderate score across all the different sociodemographic factors, Table 1.

Table 1. Summative Scores for EAC per demographic characteristics.

\begin{tabular}{|c|c|c|c|c|c|c|}
\hline \multicolumn{2}{|c|}{ Demographic Characteristics } & \multicolumn{3}{|c|}{ EAC-BF Scale Statistic Scores } & \multicolumn{2}{|l|}{ Tests } \\
\hline Factors & Categories & n & Mean & S D & $\mathbf{F}$ & Sig \\
\hline \multirow{2}{*}{ Year of Study } & 1st Year & 1991 & 149.06 & 16.1 & \multirow{2}{*}{87.562} & \multirow{2}{*}{$.000 * *$} \\
\hline & 2nd Year & 1496 & 155.88 & 21.4 & & \\
\hline \multirow{3}{*}{ Age Group } & $20 \&$ below & 609 & 153.25 & 18.88 & \multirow{3}{*}{0.704} & \multirow{3}{*}{0.495} \\
\hline & $21-25$ & 1658 & 152.8 & 19.74 & & \\
\hline & Over 25 & 510 & 151.89 & 19.21 & & \\
\hline Gender & Female & 1466 & 166.77 & 14.29 & \multirow{2}{*}{3835.063} & \multirow{2}{*}{$.000 * *$} \\
\hline \multirow{3}{*}{ Religion } & Christian & 2665 & 152.84 & 19.49 & & \\
\hline & Muslim & 82 & 150.26 & 18.57 & \multirow[t]{2}{*}{1.065} & \multirow[t]{2}{*}{0.345} \\
\hline & Other & 29 & 149.69 & 16.5 & & \\
\hline
\end{tabular}




\begin{tabular}{llllll}
\hline Demographic Characteristics & & EAC-BF Scale Statistic Scores & & Tests \\
\hline Factors & Categories & n & Mean & S D & F \\
\hline & Married & 597 & 157.62 & 18.71 & \\
Marital Status & Widowed & 2 & 169 & 26.87 & 18.395 \\
& Divorced/Separated & 20 & 169.45 & 18.05 & $.000^{* *}$ \\
Ever Taught before coming to & Not Married & 2158 & 151.3 & 19.41 & \\
college & Yes & 1173 & 160.42 & 20.11 & 358.95 \\
\hline
\end{tabular}

Significant variations were indicated among the year of study, gender, marital status and whether ever taught before coming to college.

\subsection{EAC-BF Factors Mean Scores Based on Demographic Characteristics}

Significant variations $(\mathrm{p}=0.000)$ were indicated in all factors in the following demographic characteristics, year of study, gender, marital status and whether ever taught before coming to college.

Table 2. Presents EAC-BF Factors Mean Score Based on Demographic Characteristics.

\begin{tabular}{|c|c|c|c|c|c|c|c|c|c|}
\hline & & \multicolumn{2}{|c|}{$\begin{array}{l}\text { Personal } \\
\text { Commitments }\end{array}$} & \multicolumn{2}{|c|}{ Counselor Expertise } & \multicolumn{2}{|c|}{ Facilitative Conditions } & \multicolumn{2}{|c|}{ Realism } \\
\hline & & Mean & SD & Mean & SD & Mean & SD & Mean & SD \\
\hline \multirow{4}{*}{ Age groups } & 20 Years $\leq$ & 3.23 & .36 & 3.19 & .45 & 3.21 & .37 & 3.22 & .41 \\
\hline & $21-25$ Years & 3.25 & .34 & 3.23 & .46 & 3.23 & .35 & 3.24 & .40 \\
\hline & Over 25 years & 3.23 & .35 & 3.22 & .45 & 3.22 & .36 & 3.22 & .41 \\
\hline & & \multicolumn{2}{|c|}{$\mathrm{F}=.828 \mathrm{p}=.437$} & \multicolumn{2}{|c|}{$\mathrm{F}=1.820 \mathrm{p}=.162$} & \multicolumn{2}{|c|}{$\mathrm{F}=1.277 \mathrm{p}=.279$} & \multicolumn{2}{|c|}{$\mathrm{F}=1.307 \mathrm{p}=.271$} \\
\hline \multirow{3}{*}{ Gender } & Female & 3.43 & .28 & 3.42 & .39 & 3.42 & .28 & 3.43 & .33 \\
\hline & Male & 3.03 & .29 & 2.99 & .41 & 3.01 & .30 & 3.02 & .37 \\
\hline & & \multicolumn{2}{|c|}{$\mathrm{F}=1396.596 \mathrm{p}=.000$} & \multicolumn{2}{|c|}{$\mathrm{F}=791.575 \mathrm{p}=.000$} & \multicolumn{2}{|c|}{$\mathrm{F}=960.078 \mathrm{p}=.000$} & \multicolumn{2}{|c|}{$\mathrm{F}=1388.938 \mathrm{P}=.000$} \\
\hline \multirow{3}{*}{ Year of Study: } & 1st Year & 3.10 & .34 & 3.09 & .46 & 3.09 & .34 & 3.10 & .41 \\
\hline & 2nd Year & 3.35 & .31 & 3.33 & .42 & 3.34 & .33 & 3.35 & .36 \\
\hline & & \multicolumn{2}{|c|}{$\mathrm{F}=404.489 \mathrm{P}=.000$} & \multicolumn{2}{|c|}{$\mathrm{F}=208.976 \mathrm{p}=.000$} & \multicolumn{2}{|c|}{$\mathrm{F}=383.798 \mathrm{p}=.000$} & \multicolumn{2}{|c|}{$\mathrm{F}=283.200 \mathrm{p}=.000$} \\
\hline \multirow{4}{*}{ Religion } & Christian & 3.24 & .35 & 3.22 & .45 & 3.22 & .36 & 3.23 & .40 \\
\hline & Muslim & 3.20 & .35 & 3.19 & .48 & 3.22 & .39 & 3.22 & .41 \\
\hline & Other & 3.19 & .30 & 3.14 & .47 & 3.21 & .36 & 3.20 & .35 \\
\hline & & \multicolumn{2}{|c|}{$\mathrm{F}=.878 \mathrm{p}=.416$} & \multicolumn{2}{|c|}{$\mathrm{F}=.638 \mathrm{p}=.529$} & \multicolumn{2}{|c|}{$\mathrm{F}=.161 \mathrm{P}=.851$} & \multicolumn{2}{|c|}{$\mathrm{F}=.035 \mathrm{P}=.965$} \\
\hline \multirow{5}{*}{ Marital Status } & Married & 3.30 & .34 & 3.30 & .45 & 3.29 & .35 & 3.29 & .39 \\
\hline & Widowed & 3.55 & .00 & 4.06 & .55 & 3.43 & .40 & 3.58 & .49 \\
\hline & Divorced or Separated & 3.35 & .36 & 3.42 & .50 & 3.34 & .37 & 3.39 & .37 \\
\hline & Not Married & 3.22 & .35 & 3.20 & .45 & 3.21 & .36 & 3.22 & .40 \\
\hline & & \multicolumn{2}{|c|}{$\mathrm{F}=14.659 \mathrm{p}=.000$} & \multicolumn{2}{|c|}{$\mathrm{F}=15.145 \mathrm{P}=.000$} & \multicolumn{2}{|c|}{$\mathrm{F}=13.948 \mathrm{p}=.000$} & \multicolumn{2}{|c|}{$\mathrm{F}=8.998 \mathrm{P}=.000$} \\
\hline \multirow{3}{*}{$\begin{array}{l}\text { Ever taught } \\
\text { before? }\end{array}$} & Yes & 3.28 & .34 & 3.26 & .46 & 3.28 & .35 & 3.28 & .40 \\
\hline & No & 3.21 & .35 & 3.19 & .45 & 3.19 & .35 & 3.20 & .40 \\
\hline & & \multicolumn{2}{|c|}{$\mathrm{F}=30.989 \mathrm{p}=.000$} & $\mathrm{~F}=13.9$ & $=.000$ & $\mathrm{~F}=43.0$ & & $\mathrm{~F}=27.8$ & $=.000$ \\
\hline & Village & 3.23 & .35 & 3.21 & .45 & 3.22 & .36 & 3.23 & .40 \\
\hline I grew up in & Town & 3.29 & .35 & 3.27 & .45 & 3.25 & .37 & 3.26 & .41 \\
\hline & & $\mathrm{F}=2.72$ & & $\mathrm{~F}=5.18$ & .023 & $\mathrm{~F}=2.01$ & & $\mathrm{~F}=8.56$ & 003 \\
\hline
\end{tabular}

\section{Discussion}

This study population indicated medium (neutral) expectations about counseling. These results are consistent with other research work, [20,21]. Further analysis indicated significant variation in year of study, gender, marital status, and whether they ever taught before coming to college. However, religion and whether grew up in a village or a town indicated a significance in the control group and the experimental group respectively.

\subsection{Expectations About Counseling Summative Score per Demographic Characteristics}

Significant variation in gender was as a result of a higher summative mean score among the female participants; who held more favorable expectations. This finding is in line with other findings in the general population and college students, $[17,18,20,22]$. As expected, the second years indicated more favorable expectations about counseling, due to the curriculum coverage. This is in line with other studies among undergraduate university students [23].

The married reported more accepting tendencies to counseling compared to the others. However, there was no study to compare this result within literature. This finding is attributed to the idea that the married may have undergone some counseling at some time in their lives especially during marriage either in traditional marriage or Christian and Muslim marriages, which is common among Kenyan population. Having had an experience of counseling has been found to contribute to the likelihood of individuals to have more accepting attitudes towards counseling, [23].

Those who had taught before coming to college indicated 
more favorable expectations towards counseling. There was no study to compare this result with; however this finding has been attributed to teaching experience untrained teachers get while teaching even before coming to college as discussed earlier.

\subsection{Expectations About Counseling per OMI Factors Based on Demographic Characteristics}

Expectations about counseling per factor used three factors of Personal Commitments, Counselor Expertise, and Facilitative Conditions. These were the factors identified to have been validated and indicate the actual expectations about counseling, Tinsley in his write up to this researcher indicated that using these three factors was good enough to measure expectations about counseling.

In Personal Commitment to Counseling for a mental health problem, significant variation was found in gender, year of study, marital status and whether participants ever taught before coming to college. Female participants reported more favorable commitment to counseling. This finding is consistent with other findings, [24, 25]. Those who reported being in the second year of study indicated a higher personal commitment to counseling. This has been attributed to TTCs curriculum, which may have exposed this group to more favorable expectations of counseling. Participants who had taught before coming to college indicated a higher personal commitment.

In Counselor Expertise, significant variation was indicated in gender, year of study, marital status, whether ever taught before coming to college and whether participants ever taught before coming to college and whether participants grew up in a village or town. Female trainees reported higher expectations in counselor expertise. This finding is consistent with other findings, [8, 9, 25, 26]. Second-year trainees indicated more favorable expectations about counseling. This is attributed to curriculum coverage discussed earlier. Those who reported being divorced, separated or widowed indicated more favorable expectations in this factor. This finding is attributed to the likelihood that they may have gone through some counseling while going through divorce, separation or widowhood experiences. Those who had taught before coming to college reported a higher expectation. This is consistent with what has already been discussed above about teaching experience.

In Facilitative Conditions, significant variation was indicated in gender, year of study, marital status, whether participants ever taught before coming to college and whether they grew up in a village or a town. The female teacher trainees reported higher expectations on the facilitative conditions. This finding is consistent with other research [20, 21, 28]. The widowed separated and divorced indicated a higher score than the married and unmarried. This finding is speculated to be due to the counseling those who had gone through a divorce, separation or widowhood may have gone through and they may have found counselors to be experts.

\section{Conclusion}

We conclude that expectations about counseling for a mental related problem among teacher trainees in Kenya is more unfavorable. Barriers to seeking help from college students has been implicated to include lack of knowledge of in both available services and symptoms of psychological distress, [29] We are therefore suggesting that in order to improve these expectations, psychoeducation on both mental illness and expectations about counseling should be given to the students. Furthermore adding to the training curriculum of teachers should do a continuous education on both. However, there is more research needed on an intervention, especially psychoeducation on what to expect in counseling among individuals with mental illness to determine the effectiveness.

\section{Acknowledgement}

We want to acknowledge the African Mental Health Foundation (AMHF) and Malel Bibwob Clinical Psychological Consultants Ltd. (MICAP) for the contribution they gave in terms of accessing the Internet and the journals used in the preparation of this paper. This research was not funded,

\section{References}

[1] Kessler, R. C., Aguilar-Gaxiola, S., Alonso, J., Chatterji, S., Lee, S., Ormel, J.,... \& Wang, P. S. (2009). The global burden of mental disorders: an update from the WHO World Mental Health (WMH) surveys. Epidemiologia e psichiatria sociale, $18 \quad(01), \quad 23-33$. http://dx.doi.org/10.1017/S1121189X00001421

[2] Whiteford, M. D., Degenhart, L., Rehm, J., Baxterm A. J., Ferrari, A. J. Erskine, H. E. et al. (2013). Global burden of disease attributable to mental and substance use disorders: finding from the Global Burden of Disease Study 2010, 382(9904): 1575-1586. DOI: http://dx.doi.org/10.1016/S01406736(13)61611-6

[3] Viner, R. M., Ozer, E. M., Denny, S., Marmot, M., Resnick, M., Fatusi, A., \& Currie, C. (2012). Adolescence and the social determinants of health. The Lancet, 379 (9826), 16411652. doi: 10.1016/S0140-6736(12)60149-4

[4] Chan, M. (2010). Mental health and development: targeting people with mental health conditions as a vulnerable group. World Health Organization.

[5] Gore, F. M., Bloem, P. J., Patton, G. C., Ferguson, J., Joseph, V., Coffey, C.,... \& Mathers, C. D. (2011). Global burden of disease in young people aged 10-24 years: a systematic analysis. The Lancet, 377 (9783), 2093-2102. doi: 10.1016/S0140-6736(11)60512-6

[6] Murray, C. J., Vos, T., Lozano, R., Naghavi, M., Flaxman, A. D., Michaud, C.,... \& Aboyans, V. (2013). Disability-adjusted life years (DALYs) for 291 diseases and injuries in 21 regions, 1990-2010: a systematic analysis for the Global Burden of Disease Study 2010. The lancet, 380 (9859), 2197-2223. doi: 10.1016/S0140-6736(12)61689-4 
[7] Rickwood, D. J., Deane, F. P., \& Wilson, C. J. (2007). When and how do young people seek professional help for mental health problems?. Medical Journal of Australia, 187 (7), S35.

[8] de Girolamo, G. I. O. V. A. N. N. I., Dagani, J., Purcell, R., Cocchi, A., \& McGorry, P. D. (2012). Age of onset of mental disorders and use of mental health services: needs, opportunities and obstacles. Epidemiology and psychiatric $\begin{array}{lllll}\text { sciences, } & 21 & (01), & 47-57 . & \text { DOI: }\end{array}$ http://dx.doi.org/10.1017/S2045796011000746

[9] Vogel, D. L., Wester, S. R., Wei, M., \& Boysen, G. A. (2005). The Role of Outcome Expectations and Attitudes on Decisions to Seek Professional Help. Journal of Counseling Psychology, 52 (4), 459. http://dx.doi.org/10.1037/00220167.52.4.459

[10] Mueser T. K., Corrigan P. W., Hilton D. W., Tanzman B., Schaub A. Generich S. et al. (2002). Illness Management and Recovery: A Review of the Research. Psychiatric Services, 53 (10): 1272-1284. http://dx.doi.org/10.1176/appi.ps.53.10.127

[11] Greenberg R. P., Constantino M. J. \& Bruce N. (2006). Are patient expectations still relevant for psychotherapy process and outcome? Clinical Psychology Review; 26 (6): 657-678. Doi: 10.1016/j.cpr.2005.03.002

[12] The government of the Republic of Kenya, Ministry of Education. (2010). Summative Evaluation of Primary School teacher education. Kenya Institute of Education

[13] Tinsley H. E., Bowman S. L., \& Ray S. B. (1988). Manipulation of expectancies about counseling and psychotherapy: Review and analysis of expectancy manipulation strategies and results. Journal of Counseling Psychology; 35 (1): 88-108.

[14] Tinsley, H. E. A. (1982). Expectations about Counseling. Unpublished Test Maual, University of Florida, Gainesville.

[15] Tinsley H. E. A., Workman K. R. and Kass, R. A. (1980). Factor analysis of the domain of client expectancies about counseling. Journal of Counseling Psychology; 27: 561-570. http://dx.doi.org/10.1037/0022-0167.27.6.561

[16] Hatchett, G. T. and Han, K. (2006), Development and evaluation of new factor scales for the expectations about counseling inventory in a college sample. J. Clin. Psychol., 62: 1303-1318. doi: 10.1002/jclp.20308

[17] Aegisdottir, S., Gerstein, L. H., \& Gridley, B. E. (2000). The factorial structure of the expectations about counseling questionnaire-brief form: Some serious questions. Measurement and Evaluation in Counseling and Development, 33 (1), 3-20.

[18] Kakhnovets, R. (2011). Relationships among personality, expectations about counseling, and help-seeking attitudes. Journal of Counseling and Development: JCD, 89 (1), 11.

[19] Al-Krenawi, A., Graham, J. R., Al-Bedah, E. A., Kadri, H. M., \& Sehwail, M. A. (2009). Cross-national comparison of Middle Eastern university students: Help-seeking behaviors, attitudes toward helping professionals, and cultural beliefs about mental health problems. Community mental health journal, 45 (1), 26-36. doi: 10.1007/s10597-008-9175-2.
[20] Chen, S. X., \& Mak, W. W. (2008). Seeking professional help: Etiology beliefs about mental illness across cultures. Journal of Counseling Psychology, $55 \quad$ (4), 442. http://dx.doi.org/10.1037/a0012898

[21] Cheryl Moore-Thomas, assistant professor, Robert Lent, professor (2007) Middle School Students' Expectations About Counseling. Professional School Counseling: April 2007, Vol. 10, No. 4, pp. 410-418. doi: http://dx.doi.org/10.5330/prsc.10.4.w6452p1v23485857

[22] Al-Darmaki F. \& Fatima R. (2003). Attitudes towards seeking professional psychological help: What really counts for United Arab Emirates University Students? Social Behaviour and Personality. Social Behavior and Personality: an international journal: An International Journal; 31 (5): 497. DOI: https://www.researchgate.net/.../23359643 $10.2224 / \mathrm{sbp} .2003 .31 .5 .497$

[23] Boyer, S. P., \& Sedlacek, W. E. (1987). Counseling expectations: Differences by gender and presenting problem. Counseling Center, University of Maryland.

[24] Hardin, S. I., \& Yanico, B. J. (1983). Counselor gender, type of problem, and expectations about counseling. Journal of $\begin{array}{llll}\text { Counseling } & \text { Psychology, } 30 & \text { (2), } & 294 .\end{array}$ http://dx.doi.org/10.1037/0022-0167.30.2.294

[25] Gonzalez, J. M., Tinsley, H. E. A., \&amp; Kreuder, K. R. (2002). Effects of psychoeducational interventions on opinions of mental illness, attitudes toward help seeking, and expectations about psychotherapy in college students. Journal of College Student Development, 43 (1), 51-63.

[26] Schaub, M., \& Williams, C. (2007). Examining the relations between masculine gender role conflict and men's expectations about counseling. Psychology of Men \& Masculinity, 8 (1), $40 . \quad \mathrm{http} / / / \mathrm{dx}$. doi.org/10.1037/15249220.8.1.40

[27] Robitschek C., Hershberger C. and Amy R. (2005). Predicting expectations about counseling: psychological factors and gender implications. (Research) Journal of Counseling and Development; 83 (4): 457-469. DOI: 10.1002/j.15566678.2005.tb00367.x

[28] Scheel M. J., Razzhavaikina T. I., Allen-Portsche S. Backhaus A. Madabhushi S. and Rudy M. (2008). International Students Expectations and knowledge of Counseling after viewing a Multicultural Video. Asian Journal of Counseling; 15 (1): 3335 .

http://www.fed.cuhk.edu.hk/en/ajc/200800150001/0033.htm

[29] Sharon Huixian Lu, Blake Farran Dear, Luke Johnston, Bethany May Wootton, Nickolai Titov (2014). An Internet Survey of Emotional Health Treatment Seeking and Barriers to Accessing Mental Health Treatment among ChiniseSpeaking international Students in Australia. Counseling $\begin{array}{llll}\text { Psychology } & \text { Quarterly, } 27 & \text { (1): } & \text { DOI: }\end{array}$ 10.1080/09515070.2013.824408 